\title{
Congenital Hypothyroidism Long-Term Follow-up Project: Navigating the Rough Waters of a Multi-Center, Multi-State Public Health Project
}

\author{
Kupper Wintergerst • Gina Gembel • Tracey Kreipe • Patrick Zeller • Erica Eugster • \\ Bill Young • Karen Andruszewski • Mary Kleyn • Troi Cunningham • \\ Sandy Fawbush • Nancy Vanderburg • Joe Sockalosky • Ram Menon • \\ Sharon Linard • Gary Hoffman • Lisa Gorman
}

Received: 14 February 2014 / Accepted: 28 October 2014 / Published online: 18 November 2014

(C) National Society of Genetic Counselors, Inc. 2014

\begin{abstract}
The Region 4 Midwest Genetics Collaborative, made up of seven regional states (Illinois, Indiana, Kentucky, Michigan, Minnesota, Ohio, and Wisconsin), brought together pediatric endocrinologists, state laboratory experts, public health follow-up specialists, and parents of children with congenital hypothyroidism $(\mathrm{CH})$ to identify the three-year follow-up management and education patterns of primary care clinicians and pediatric endocrinologists in the care of children diagnosed with $\mathrm{CH}$ by state newborn screening (NBS) programs. Among a number of challenges, each state had different NBS methods, data systems, public health laws, and institutional review board (IRB) requirements. Furthermore,
\end{abstract}

\footnotetext{
K. Wintergerst $(\bowtie)$

Department of Pediatrics, Endocrinology, School of Medicine,

University of Louisville, Louisville, KY, USA

e-mail: kawint01@louisville.edu

G. Gembel • L. Gorman

Region 4 Genetics Collaborative, Michigan Public Health Institute, Okemos, MI, USA

K. Wintergerst $\cdot$ T. Kreipe $\cdot$ P. Zeller $\cdot$ E. Eugster $\cdot$ B. Young $\cdot$

K. Andruszewski $\cdot$ M. Kleyn $\cdot$ T. Cunningham $\cdot$ S. Fawbush •

N. Vanderburg $\cdot$ J. Sockalosky $\cdot$ R. Menon $\cdot$ S. Linard $\cdot$ G. Hoffman

Endocrine Workgroup, Region 4 Genetics Collaborative, Okemos,

MI, USA

T. Kreipe

Illinois Department of Public Health, Springfield, IL, USA

P. Zeller

Academic Endocrinology Metabolism \& Nutrition, Inc., Wheaton,

IL, USA

E. Eugster

Indiana University School of Medicine, Indianapolis, IN, USA
}

the diagnosis of $\mathrm{CH}$ was complicated by the timing of the NBS sample, the gestational age, weight, and co-morbidities at delivery. There were 409 children with $\mathrm{CH}$ identified through NBS in 2007 in the seven state region. The clinician of record and the parents of these children were invited to participate in a voluntary survey. Approximately $64 \%$ of clinician surveys were collected with responses to questions relating to treatment, monitoring practices, educational resources, genetic counseling, and services provided to children with confirmed $\mathrm{CH}$ and their families. Nearly one-quarter ( $24 \%$ ) of parents surveyed responded to questions relating to treatment, education, genetic counseling, resources, and

B. Young $\cdot$ K. Andruszewski $\cdot$ M. Kleyn

Michigan Department of Community Health, Lansing, MI, USA

T. Cunningham $\cdot$ S. Fawbush

Kentucky Department for Public Health, Frankfort, KY, USA

N. Vanderburg

Minnesota Department of Health, St. Paul, MN, USA

J. Sockalosky

Children's Hospitals and Clinics of Minnesota, St. Paul, MN, USA

R. Menon

University of Michigan C.S. Mott Children's Hospital, Ann Arbor, MI, USA

S. Linard

Ohio Department of Health Laboratory, Reynoldsburg, OH, USA

G. Hoffman

Wisconsin State Laboratory of Hygiene, Madison, WI, USA 
services they received or would like to receive. De-identified data from six of the seven states were compiled for analysis, with one state being unable to obtain IRB approval within the study timeline. The data from this collaborative effort will improve state follow-up programs and aid in developing three-year follow-up guidelines for children diagnosed with $\mathrm{CH}$. To aid in the facilitation of similar public health studies, this manuscript highlights the challenges faced, and focuses on the pathway to a successful multi-state public health endeavor.

Keywords Congenital hypothyroidism $\cdot$ Public health . Long-term follow-up

\section{Introduction}

Newborn screening (NBS) for congenital disorders is an essential public health system intended to reduce death and disability in newborns with a wide range of genetic conditions. The utility of screening for congenital hypothyroidism $(\mathrm{CH})$ was first demonstrated in the early 1970 s (Klein et al. 1974). As the benefits of early diagnosis and treatment of $\mathrm{CH}$ were established, it was gradually adopted into NBS throughout the United States and is now mandated or recommended in all 50 states; the District of Columbia; and U.S. territories, which include America Samoa, Guam, Northern Mariana Islands, Puerto Rico, and the U.S. Virgin Islands. (NNSGRC 2011; US Task Force 1996, 2008). Approximately, 1,900 infants with $\mathrm{CH}$ in the United States are detected annually (NNSGRC 2006). Recent research demonstrates that, while previously thought of as a predominantly sporadic disease leading to dysgenesis (85-90\% of cases), there are distinct genetic forms of isolated or syndromic thyroid dysgenesis. These findings, while supporting nonMendelian modes of inheritance, have helped to further elucidate the genetic basis of this condition (Szinnai 2014). Regardless of etiology, though, infants must be quickly and properly identified and treated in order to prevent permanent cognitive and physical delays. In addition, clinical follow-up by an endocrinologist for the first three years of life, if not lifelong, is essential to ensure that these children receive appropriate monitoring and management. The American Academy of Pediatrics (AAP) and the European Society for Pediatric Endocrinology (ESPE) provide guidelines for the treatment of $\mathrm{CH}$. These guidelines include a recommendation for follow-up of $\mathrm{CH}$ cases until at least three years of age to distinguish permanent versus transient disease states (Rose and Brown 2006; ESPE Guidelines 1999).

The immediate impact on the health of infants diagnosed with $\mathrm{CH}$ by NBS is undisputed, yet little follow-up data are available assessing the long-term effectiveness of the program at the state, regional, or national level. It is important to note that some parents of children with $\mathrm{CH}$ choose to discontinue thyroid hormone replacement without appropriate medical advice and are lost to follow-up (Kemper et al. 2010; Korzeniewski et al. 2013). The Health Resources and Services Administration (HRSA)-funded Region 4 Midwest Genetics Collaborative brought together pediatric endocrinologists, state laboratory personnel, public health follow-up specialists, and parents of children with $\mathrm{CH}$ to better understand followup for patients with $\mathrm{CH}$. The Region 4 Midwest Genetics Collaborative (Region 4), which includes Illinois, Indiana, Kentucky, Michigan, Minnesota, Ohio, and Wisconsin, strives to improve the health of children and their families by promoting the translation of genetic medicine into public health and health care services. The Region 4 Midwest Genetics Collaborative continues to utilize the infrastructure that launched projects like the Laboratory Performance Database Region 4 Stork (R4S) (Fleischman et al. 2014; Hall et al. 2014; McHugh et al. 2011) and the Inborn Errors of Metabolism Information System (IBEM-IS) (Berry et al. 2010). As the vanguard for a regional initiative to develop formal longterm follow-up guidelines, Region 4 established the Congenital Hypothyroidism 3-year Follow-up Workgroup (R4 CH Workgroup) in 2011.

The R4 CH Workgroup sought to identify the management, genetic counseling, and medical education patterns of pediatric endocrinologists and primary care providers for the care of children diagnosed with $\mathrm{CH}$ by state NBS programs within the seven state region. Specifically, the $\mathrm{R} 4 \mathrm{CH}$ Workgroup sought to identify management practices for re-evaluating the diagnosis of $\mathrm{CH}$ at or before three years of age. To accomplish this, the Workgroup overcame a variety of logistical, regulatory, and legal challenges to successfully complete the study. Among a number of these challenges, each state had different NBS methods, data systems, public health laws, and institutional review board (IRB) requirements. Despite these barriers, the data obtained from this collaborative effort will be used to improve state follow-up programs within the region and aid in developing threeyear follow-up guidelines for children diagnosed with $\mathrm{CH}$ nationwide. To aid in the facilitation of similar public health studies, this manuscript highlights the challenges faced and focuses on the pathway to a successful multi-state collaborative endeavor.

\section{Materials and Methods}

Regional Leadership and State Participants

The study was developed and executed under the guidance of the Region 4 Midwest Genetics Collaborative and utilized the 
infrastructure provided by the HRSA grantee, the Michigan Public Health Institute (MPHI) (Fig. 1). The Office of Research Integrity at MPHI approved the study protocol. Representatives from each state in the region were included in the $\mathrm{CH} 3$-year Follow-up Workgroup. While the $\mathrm{CH}$ Workgroup met face-to-face during the Region 4 Genetics Collaborative Regional Meeting, the majority of the study protocol and materials were developed during monthly teleconferences, webinars and through email communique. Multi-state participation was crucial to the success of the project to ensure sufficient sample size, a range of responses, and a nuanced comparative analysis. A Project Lead from the $\mathrm{CH}$ Workgroup was chosen to direct the study, but State Leads were also necessary in order to focus the effort within the particular state and to maintain momentum for completion of the study. State Leads were identified at the outset of the study based on their role within the state NBS program or in managing children with $\mathrm{CH}$, and their willingness to commit time and effort to the project.

\section{Subjects}

The inclusion criterion for parent and provider participation in the study was a diagnosis of $\mathrm{CH}$ through state NBS and a birth date between January 1, 2007 through December 31, 2007. Of note, the NBS blood sample for each subject was required to have been obtained within the first 48 hours of life, as states have varying collection protocols for children requiring support, such as total parenteral nutrition and blood products, which may affect $\mathrm{CH}$ screening results. State variations also include differing protocols or provider decisions regarding repeat NBS blood samples or serum draws for specific congenital disorders for confirmatory testing. As this was a survey study, the diagnosis of $\mathrm{CH}$ was purely based on NBS records and survey responses, and direct medical records were not reviewed. Children born in 2007 were chosen to allow for at least three years since diagnosis by NBS before study participation. The NBS record contains demographic data such as the child's name, date of birth, mother's name, maternal address and phone number provided at birth, and the clinician identified at birth as being responsible for the child's care. A separate record contains the child's positive NBS test(s) result tied to the dried blood spot sample. After obtaining IRB approval, the state laboratory and/or health department accessed its own records to identify children who met the inclusion criteria.

\section{Study Design}

The study was a survey collection with two mirrored surveys, one for clinicians and one for families (Fig. 2). Pediatric endocrinologists, state laboratory personnel, and public health follow-up specialists in the Region $4 \mathrm{CH}$ 3- year Follow-up
Workgroup met regularly to discuss, develop, and revise survey instruments. In addition, the Workgroup sought and received input from parents of children diagnosed with $\mathrm{CH}$ for study and survey design. The Clinician Survey used in this study was adapted following a $2009 \mathrm{CH}$ pilot study performed by our Region 4 colleagues from Michigan (Korzeniewski et al. 2013). The Clinician Survey includes questions relating to treatment, monitoring practices, educational resources, genetic counseling, and services provided to the child with $\mathrm{CH}$ and his/her family.

The Parent Survey was developed by the CH Workgroup with the goal of understanding parent preferences for educational resources and reasons children were lost to follow-up. It included questions relating to treatment, educational resources, genetic counseling, and services parents received, or would like to receive, from their state's health department as well as questions pertaining to diagnosis and treatment of $\mathrm{CH}$.

\section{Protocol and Study Instrument Development}

To streamline protocol and study instrument development, the Project Lead was responsible for developing the core protocol draft design, followed by detailed review by the State Leads and additional members of the $\mathrm{CH}$ Workgroup. Core data elements within the surveys could not be altered so that data integrity was maintained. Staff from MPHI worked with State Leads to obtain IRB approval within their respective states as well as to ensure that the overall protocol met federal requirements for protection of human subjects. Individual states worked within their own systems to meet their institutional IRB requirements and/or state laws as applicable. Each state laboratory and/or public health department identified children with $\mathrm{CH}$ from the state NBS database, which included records that were stored either electronically or in a hard copy. As a multi-state public health project, we were cognizant that the general design would have to be relatively basic and the protocol language would have to allow for some flexibility for each State Lead to receive IRB approval. The number of states involved and the variability in NBS program practices ultimately resulted in several protocol modifications before receiving $\mathrm{CH}$ Workgroup approval.

Each state health department mailed a cover letter with informed consent, Clinician Survey, and self-addressed stamped return envelope to the clinician identified through the procedure noted above. Two weeks after the initial survey distribution, each state health department mailed a second copy of the Clinician Survey and return envelope to nonrespondents. Two weeks after the second survey distribution, each state health department called clinician non-respondents to provide a verbal request to complete and return the survey. If a different managing clinician was identified either in the 
Fig. 1 Region 4 genetics collaborative - $\mathrm{CH}$ 3-year followup

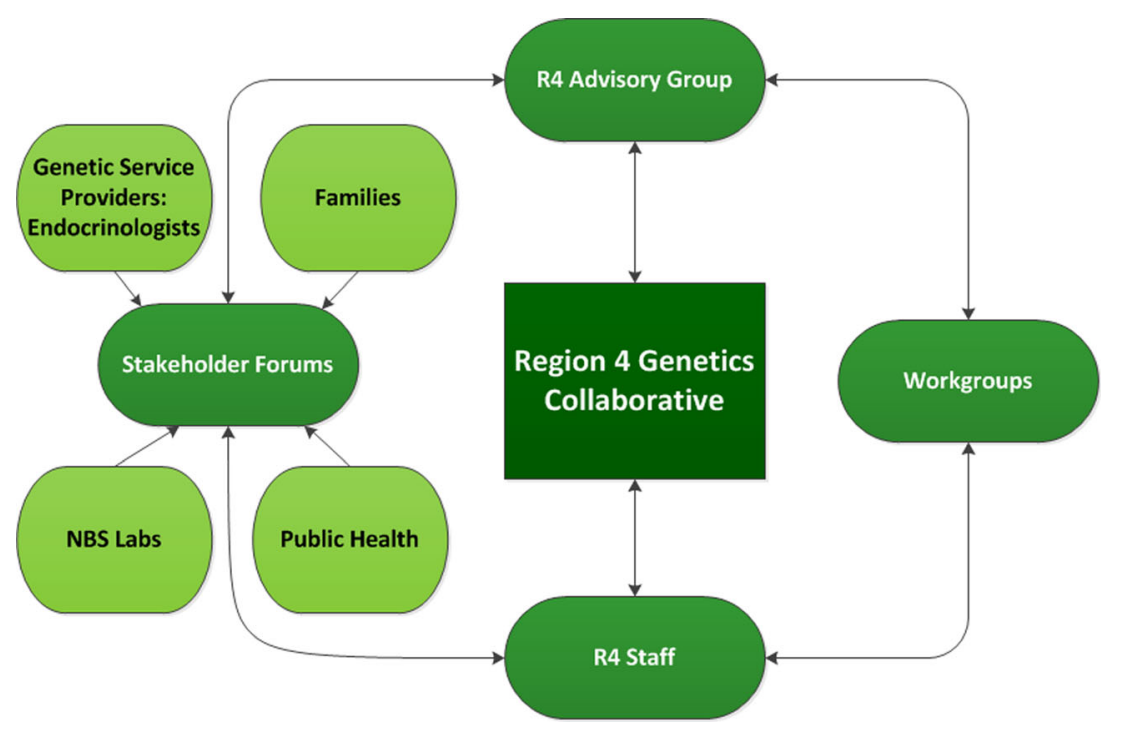

returned survey or phone call, the state health department mailed a new Clinician Survey to that identified clinician.

A similar, but abbreviated, method was used for the Parent Survey distribution. Depending on their IRB approval, each state health department mailed a cover letter with informed consent, Parent Survey, and self-addressed stamped return envelope to the parent identified through the procedure noted above. Two weeks after the initial survey distribution, state health departments mailed a second copy of the Parent Survey and return envelope to non-respondents. Two states sent the

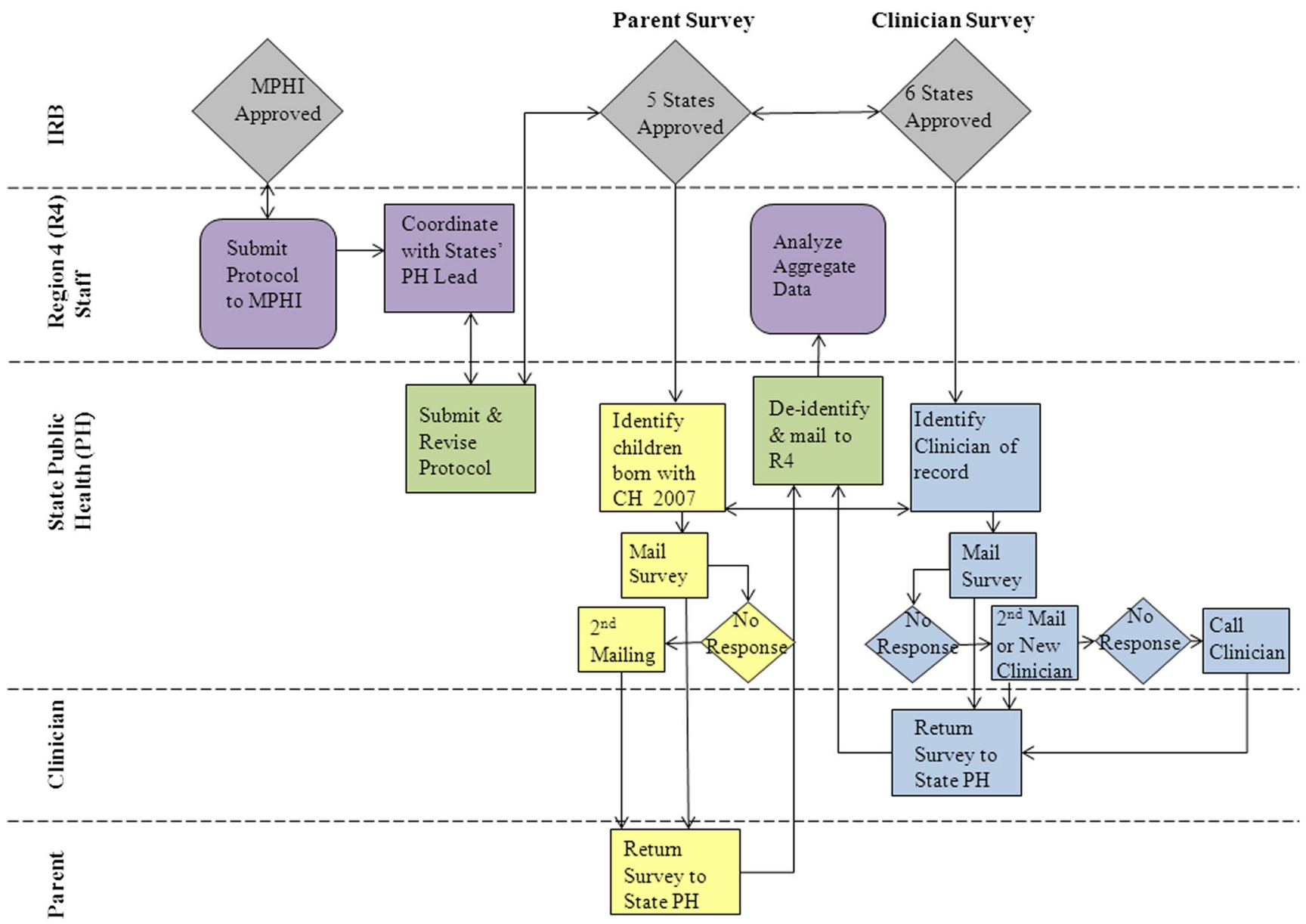

Fig. 2 Flow chart of study design \& collaborative effort 
parent survey via certified mail so that they were able to ascertain whether or not the parent had received the survey and were non-respondent or if the parent no longer lived at the address of record. The first Clinician and Parent Surveys for any state were mailed in 2012.

The Clinician and Parent Surveys had a cover page which included the subject's name and date of birth. This cover page assisted the state's health department in tracking survey responses. When the surveys were returned, the state health department removed the cover page containing the respondent's identifying information. The state health department assigned an identification (ID) code to unique to the survey. The ID code was used in lieu of the subject's name on all study data to link clinician and parent responses, where applicable. The de-identified data were sent to MPHI for analysis.

\section{Data Analysis}

De-identified data were sent from each state to the Region 4 headquarters at MPHI for aggregate analysis. De-identified raw data were received on a rolling basis from six of seven states. A record was entered for each unique ID code, allowing for paired surveys from clinicians and parents to be matched within the same record. Once data collection was complete, an MPHI epidemiologist used statistical software to analyze the data, and survey responses were reported out to $\mathrm{CH}$ Workgroup members for review and discussion. Additional data analysis was completed upon discussion with the Workgroup based on initial review.

\section{Results}

\section{General}

Overall, the study took 26 months from the initial $\mathrm{CH}$ Workgroup meeting to the completion of the aggregate data analysis (Fig. 3). Although study development began in early 2011, the first surveys were not mailed until 2012. The $\mathrm{CH}$ Workgroup met in person twice and by teleconference 21 times with additional email communications between members throughout the duration of the study. Development of the study protocol and data collection elements was performed in seven months, with all members of the $\mathrm{CH}$ Workgroup providing input. The general study protocol required 11 revisions, including nine Clinician Survey and seven Parent Survey revisions. Revisions ranged from simple grammatical adjustments for improved responder comprehension to the removal and addition of significant data elements determined by the $\mathrm{CH}$ Workgroup. Including the MPHI IRB approval for overall study responsibilities and, specifically, for Michigan participation, five of the seven states sought and received IRB approval from their respective Health Departments. For those states obtaining IRB approval, it was received within a mean of 4.6 months (range, $1-13$ months). An additional state collected data elements of interest as part of their long-term follow-up program, and, for this state, MPHI was able to submit a request to obtain de-identified long-term follow-up data elements from their long-term follow-up program for children born in 2007. One state encountered barriers obtaining IRB approval through their NBS program due to state laws restricting data access to public health records and allowances to contact health care providers and families. For this reason, no data were ultimately collected from this state for the study. In total, the $\mathrm{CH}$ workgroup was able to aggregate data from six of the seven states in Region 4.

\section{Survey and Data Collection}

A total of 409 children were identified in Region 4 as having been diagnosed with $\mathrm{CH}$ in 2007. This resulted in a combined birth prevalence of 1:1,836 for the seven states in Region 4 (Table 1). For those states receiving IRB approval, Clinician and Parent Surveys were mailed according to their approved contact protocol. Aside from the self-addressed stamped envelopes, no other incentive for the return of the Clinician and Parent Survey was used. One of the six participating states did not have access to parent names and addresses, so only clinician survey responses were collected. Overall, this allowed clinician data collection on $37.9 \%$ and parent data collection on $18.8 \%$ of the diagnosed infants in Region 4 for this year. Of the 409 infants identified as having been diagnosed with $\mathrm{CH}$, Clinician Surveys were mailed for 334 unique patients

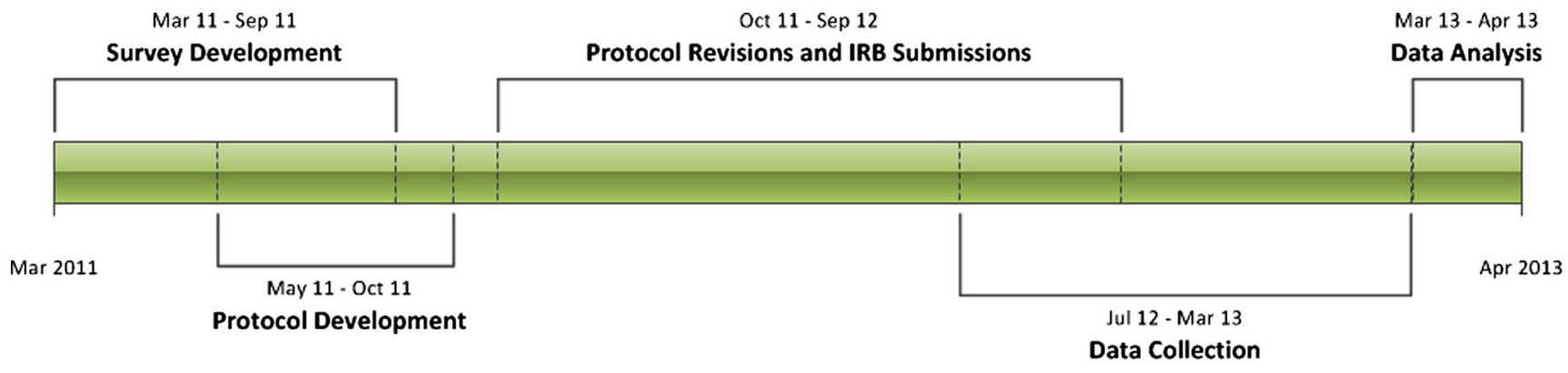

Fig. $3 \mathrm{CH}$ 3-year follow-up study timeline 
Table 1 Region 4 NBS CH birth prevalence for 2007

\begin{tabular}{llrrr}
\hline State & NBS 1st tier screening method & Live births & CH diagnosis & Incidence \\
\hline Illinois & TSH & 180,530 & 94 & $1: 1,920$ \\
Indiana & TSH & 89,719 & 42 & $1: 2,136$ \\
Kentucky & T4/TSH & 58,507 & 24 & $1: 2,438$ \\
Michigan & TSH & 125,172 & 85 & $1: 1,473$ \\
Minnesota & TSH & 73,675 & 46 & $1: 1,602$ \\
Ohio & TSH & 150,784 & 75 & $1: 2,010$ \\
Wisconsin & TSH & 72,757 & 43 & $1: 1,692$ \\
Total & & 751,144 & 409 & $1: 1,836$ \\
\hline
\end{tabular}

NBS Newborn Screening, $\mathrm{CH}$ Congenital Hypothyroidism

with a response rate of $63.7 \%$; parent surveys were mailed for 291 patients with $24.4 \%$ returned (Table 2 ). Survey collection took a mean of seven months after initial disbursement (range, 1-8 months). We received paired Clinician and Parent Surveys for the same infant on $14 \%$ of surveys returned.tgrouptgroup

\section{Discussion}

With 751,144 live births in Region 4 during 2007, we anticipated identifying approximately 380 children whose NBS was positive for $\mathrm{CH}$. This was based on $2006 \mathrm{NBS}$ data demonstrating a national birth prevalence of 1:2,209 and a regional birth prevalence of 1:1,972 (NNSGRC 2006). However, 409 children in our region were confirmed to have $\mathrm{CH}$ in 2007 , indicating a birth prevalence of 1:1,836 (range, 1:1,472-2,438). Demographic differences, testing methods (TSH versus TSH/T4), and state-specific cutoff values are likely to be among the major reasons for this variability. The diagnostic sensitivity was also potentially influenced by the timing of the first NBS blood sample, gestational age, weight of the infant, use of certain therapies, such as blood products, and infant co-morbidities. While infants with only transient abnormalities with their thyroid function studies were likely included, this very fact reinforces the importance of reevaluating the diagnosis at or before three years of age.

While the time to study completion was significant, we successfully retrieved unique survey data on $37.9 \%$ of infants born with $\mathrm{CH}$. To our knowledge, this is the first multi-state study assessing NBS long-term follow-up factors related to clinical management and education practices of $\mathrm{CH}$.

The two greatest challenges in completing this study included the development of complimentary mirrored Clinician and Parent Surveys and ensuring that all state and federal privacy laws and Health Insurance Portability and Accountability Act (HIPAA) requirements were followed. This would not have been possible without state laboratory and health department representative involvement in the $\mathrm{CH}$ Workgroup. These representatives were knowledgeable about state regulations governing the access of public health data for this purpose. In addition, they provided valuable input during survey development. The creation of surveys that provided us with informed data was complex and difficult. All members of the $\mathrm{CH}$ Workgroup, including clinicians, public health personnel, and parent volunteers, were required to provide their input. The Clinician Surveys were then vetted by clinician volunteers, working with $\mathrm{CH}$ Workgroup members, using fictitious patients with varying backgrounds and treatment plans. The Parent Surveys were vetted using a convenience sample of parent volunteers to evaluate adaptability, ease of use, and to address any evident potential literacy or cultural issues. The majority of the time for protocol design was dedicated to refining the surveys. As the validity of this form of study is dependent on the data collected from the surveys, we would strongly recommend a similar design model for comparable studies in other regions (Table 3).tgroup

\section{Regulatory and Legal Issues}

As stated above, the inclusion of state laboratory and public health personnel in the $\mathrm{CH}$ Workgroup was an absolute necessity for this study to be successful. While there are particular federal privacy laws, including HIPAA, that had to be followed, each state also has specific laws governing protection of private health information. In recent years, NBS has come under greater scrutiny by the public, largely due to concerns regarding storage, consent, and use of genetic

Table 2 Survey collection statistics

\begin{tabular}{llcl}
\hline & Surveys mailed & Surveys returned & Response rate \\
\hline Clinician & $334^{\mathrm{a}}$ & 213 & $63.7 \%$ \\
Parent & $291^{\mathrm{b}}$ & 77 & $24.4 \%$ \\
\hline
\end{tabular}

a 75 clinician surveys not mailed due to lack of IRB approval

${ }^{\mathrm{b}} 118$ parent surveys not mailed due to lack of IRB approval 
Table 3 Multi-state public health project model

1) Establish Project Lead

a) Develop project outline

b) Create draft protocol

2) Centralize communication (Regional Collaborative (RC) infrastructure/fixed meeting schedule)

3) Identify key stakeholders in each state

a) Clinicians

b) Public health department personnel

c) State lab personnel

d) Parents/patients

4) Create focused workgroup with clear goals and project timeline

5) Review project details and ratify study protocol

a) Allow open forum review

b) Allow protocol flexibility in language without compromising integrity of data collection and analysis

c) Create de-identification process to ensure privacy compliance

6) Identify key differences between state laws and regulations influencing the sphere of study

7) Identify potential costs

8) Institutional Review Board (IRB) submission

a) Utilize RC infrastructure to assist

b) Discuss any necessary modifications requested

9) Perform Study

10) Utilize RC for data management and analysis

material (Botkin et al. 2012; Hendrix et al. 2013; Lewis et al. 2011). Keeping both public concerns and privacy laws in mind, Region 4 sought and received IRB approval to proceed with study activities in six of the seven states. Table 4 details state-specific program information related to the project, including that requiring any study protocol adaptations to comply with state regulations. Two states, Indiana and Minnesota, already had health department regulatory and follow-up systems in place to directly participate in the project. In Indiana, the Indiana State Health Department (ISDH), in accordance with legislation, acts as a centralized program providing diagnosis, follow-up, management, and family counseling for infants and families identified with $\mathrm{CH}$ through the NBS program. This project, therefore, fell under the ISDH's authorized scope of responsibilities. While Minnesota did have a limited follow-up system in place, they were required to obtain IRB approval, but the project was considered exempt. While we defined "long-term follow-up" for the purposes of this project as extended follow-up beyond the initial confirmed diagnosis of $\mathrm{CH}$, only Illinois' program extended beyond the first three years of life at the time of our study.tgroup

This is in contrast to the remaining four states (Illinois, Kentucky, Michigan, and Wisconsin) which required submission to a state health department or specific university IRB, depending on the operational framework of the specific state NBS program. The reason for these differences is highlighted by the variation in NBS program coordination. In comparison to Indiana, for example, the Wisconsin NBS program is administered by the Wisconsin Department of Health (WDPH) and the testing is performed at the State Laboratory of Hygiene (WSLH) which is part of the University of Wisconsin. WDPH's role is to provide policy decisions (e.g., which disorders screened for) and be responsible for the technical operations of the program such as blood collection, testing, reporting, and short-term follow-up. All NBS data (testing results) are kept at WSLH, necessitating submission for IRB approval through the University of Wisconsin. However, the University of Wisconsin does not store parent contact information (addresses or phone numbers), as this is housed by the WDPH, from which IRB approval was not sought or approved for this study, creating some difficulty with parent survey collection.

Despite a very diligent effort from $\mathrm{CH}$ Workgroup members from the Public Health Laboratory of the Ohio Department of Health, we were unable to collect data from Ohio within the required timeframe. This was due to state laws that prevented the NBS Program and laboratory access to public health data. The study protocol was later submitted and approved through the state's Title V, Children with Special

Table 4 NBS programs and the CH LTFU project by state

\begin{tabular}{|c|c|c|c|c|c|c|c|}
\hline State & $\begin{array}{l}\text { NBS program } \\
\text { coordination }\end{array}$ & $\begin{array}{l}\text { Legislation for NBS } \\
\text { program }\end{array}$ & $\begin{array}{l}\text { STFU } \\
\text { program }\end{array}$ & $\begin{array}{l}\text { LTFU } \\
\text { program }\end{array}$ & $\begin{array}{l}\text { Required protocol } \\
\text { changes }\end{array}$ & $\begin{array}{l}\text { IRB } \\
\text { submission }\end{array}$ & $\begin{array}{l}\text { Full data } \\
\text { set }\end{array}$ \\
\hline Illinois & DPH & Yes & Yes & Yes & Yes & DPH & Yes \\
\hline Indiana & DPH, UA & Yes & Yes & No & No & $\longrightarrow$ & Yes \\
\hline Kentucky & DPH, UA & Yes & Yes & No & No & DPH & Yes \\
\hline Michigan & DPH, UA & Yes & Yes & No & Yes & $\mathrm{DPH}$ & Yes \\
\hline Minnesota & $\mathrm{DPH}$, & Yes & Yes & Yes & Yes & DPH & Yes \\
\hline Ohio & DPH & Yes & Yes & No & Yes & DPH & No \\
\hline Wisconsin & DPH, UA & Yes & Yes & No & Yes & UA & No \\
\hline
\end{tabular}

NBS Newborn Screening, CH Congenital Hypothyroidism, LTFU Long-Term Follow-up ( $>1$ year), STFU Short-Term Follow-up $(<1$ year), IRB Institutional Review Board, DPH Department of Health, $U A$ University Affiliation 
Health Care Needs Program, as they have broader authority to contact their enrollees and access data from the state's Birth Defects Registry. Although we were unable to overcome these barriers for the current study, the necessary IRB approval was successfully obtained to participate in future long-term follow-up activities. This was a valuable lesson that demonstrated the importance of careful scrutiny of public health processes, and the potential need to explore alternatives when faced with challenging restrictions to study participation and data collection.

\section{Data Analysis}

The states' departments of health mailed, via certified post, only de-identified raw data to the Region 4 evaluator at MPHI to conduct all the data entry and analysis. While numerous resources for data analysis existed within each state, it was necessary to aggregate the data to strengthen the utility of the results and the regional collaborative was a neutral site for compiling multi-state data. In addition to individual state data analysis, the ongoing participation of the Region 4 evaluator allowed the $\mathrm{CH}$ Workgroup to discuss results and to begin formulating state and region-specific long-term follow-up program plans.

\section{Future Direction \& Benefits of Regional Collaboration}

Since its adoption into national public health, NBS has grown to include testing for dozens of congenital disorders. With this expansion, there is a growing need for program accountability; not simply to demonstrate the cost-benefit of NBS at the state and national level (Schoen et al. 2002), but also to develop a more standardized approach to management, genetic counseling, education, and long-term follow-up of disorders identified by NBS. The discrepancy between clinical management guidelines for the long-term follow-up of $\mathrm{CH}$ and current practices, as demonstrated by Korzeniewski et al., illustrates this importance (Korzeniewski et al. 2013).

In addition to providing a means to evaluate $\mathrm{CH}$, as it pertains to NBS, this collaborative effort provided a number of other benefits. Without exception, this project led to a significant degree of introspection within each state program. In fact, several states within Region 4, including Illinois, Indiana, Michigan, and Minnesota, have already adopted changes to improve their NBS protocols, data collection, and follow-up programs based on their experience from this project. The remaining states are all in the process of evaluating their programs to improve services.

Michigan, for example, has decided to incorporate the Clinician Survey as part of its routine follow-up and plans to use the results to more accurately estimate the birth prevalence of permanent $\mathrm{CH}$ in newborns and to reduce the number of children with $\mathrm{CH}$ who are lost-to-follow-up. Minnesota began long term follow-up in 2008 but, as a result of this study, recognized the benefit of including data elements for monitoring children regarding treatment decisions beyond the current two years of active monitoring. Furthermore, Minnesota has incorporated new knowledge into family education and now emphasizes the importance of maintaining a relationship with a specialist to continuously monitor and provide medical management for children diagnosed by NBS.

Regardless of design simplicity, multi-center, multi-state public health studies require careful planning that includes a detailed review of IRB and state law requirements. Adequate participation from public health departments and key stakeholders within the medical communities in each state is a necessity. The data from this effort will be used to improve state follow-up programs and aid in developing three years follow-up guidelines for children diagnosed with $\mathrm{CH}$. Future regional projects such as this one will provide valuable insight into the common practices of NBS programs and clinicians with best practices.

Acknowledgments Supported by HRSA MCHB U22MC03963/ H46MC24092. Special thanks to the additional members of the $\mathrm{CH}$ Workgroup: Irene Hong-McAtee, Carolyn Dunson-Dillard, Monika Chaudhari, Barb Deluka, Courtney Eddy, Eileen White, Anna Starr, Michelle Kempf-Weibel, Patricia Donohoue, Samantha Blum, Susan Rose; and to the collaborative assistance from the public health and state laboratory personnel from the Illinois Department of Public Health, Indiana State Department of Health, Kentucky Department of Health, Michigan Department of Community Health, Minnesota Department of Health, Ohio Department of Health, and Wisconsin Department of Health Services.

\section{Compliance with Ethical Standards}

Disclosure of Conflict of Interest Kupper Wintergerst does not have any conflict of interest to disclose.

Lisa Gorman does not have any conflict of interest to disclose. Tracey Kreipe does not have any conflict of interest to disclose. Patrick Zeller does not have any conflict of interest to disclose. Erica Eugster does not have any conflict of interest to disclose. Bill Young does not have any conflict of interest to disclose. Karen Andruszewski does not have any conflict of interest to disclose. Mary Kleyn does not have any conflict of interest to disclose. Troi Cunningham does not have any conflict of interest to disclose. Sandy Fawbush does not have any conflict of interest to disclose. Nancy Vanderburg does not have any conflict of interest to disclose. Joe Sockalosky does not have any conflict of interest to disclose. Ram Menon does not have any conflict of interest to disclose. Sharon Linard does not have any conflict of interest to disclose. Gary Hoffman does not have any conflict of interest to disclose. Gina Gembel does not have any conflict of interest to disclose.

Research Involving Human Participants and/or Animals All procedures performed in studies involving human participants were in accordance with the ethical standards of the institutional and/or national research committee and with the 1964 Helsinki declaration and its later amendments or comparable ethical standards. 
Informed Consent Where exemption was not applicable and obtained, informed consent was obtained from all individual participants included in the study.

\section{References}

Berry, S. A., Jurek, A. M., Anderson, C., Bentler, K., \& Region 4 Genetics Collaborative Priority 2 Workgroup. (2010). The inborn errors of metabolism information system: A project of the Region 4 Genetics Collaborative Priority 2 Workgroup. Genet Med., $12(12$ Suppl), S215-9. doi:10.1097/GIM.0b013e3181fe5d23.

Botkin, J. R., Rothwell, E., Anderson, R., Stark, L., Goldenberg, A., Lewis, M., Burbank, M., \& Wong, B. (2012). Public attitudes regarding the use of residual newborn screening specimens for research. Pediatrics, 129(2), 231-238. doi:10.1542/peds. 20110970.

European Society for Pediatric Endocrinology (ESPE). (1999). Revised guidelines for neonatal screening programmes for primary congenital hypothyroidism. Hormone Research, 52, 49-52. doi:10.1159/ 000023433

Fleischman, A., Thompson, J. D., \& Glass, M. (2014). Systematic data collection to inform policy decisions: integration of the region 4 stork (R4S) collaborative newborn screening database to improve MS/MS newborn screening in Washington State. JIMD Reproduction, 13, 15-21. doi:10.1007/8904_2013 266.

Hall, P. L., Marquardt, G., McHugh, D. M., Currier, R. J., Tang, H., Stoway, S. D., et al. (2014). Postanalytical tools improve performance of newborn screening by tandem mass spectrometry. Genetics in Medicine. doi:10.1038/gim.2014.62.

Hendrix, K. S., Meslin, E. M., Carroll, A. E., \& Downs, S. M. (2013). Attitudes about the use of newborn dried blood spots for research: a survey of underrepresented parents. Academic Pediatrics, 13(5), 451-457. doi:10.1016/j.acap.2013.04.010.

Kemper, A. R., Ouyang, L., \& Grosse, S. D. (2010). Discontinuation of thyroid hormone treatment among children in the United States with congenital hypothyroidism: findings from health insurance claims data. BMC Pediatrics, 10, 9. doi:10.1186/1471-2431-10-9.
Klein, A. H., Agustin, A. V., \& Foley, T. P. (1974). Successful laboratory screening for congenital hypothyroidism. Lancet, 2(7872), 77-79. doi:10.1016/S0140-6736(74)91637-7.

Korzeniewski, S. J., Grigorescu, V., Kleyn, M., Young, W. I., Birbeck, G., Todem, D., Romero, R., \& Paneth, N. (2013). Transient hypothyroidism at 3-year follow-up among cases of congenital hypothyroidism detected by newborn screening. Journal of Pediatrics, 162(1), 177-182. doi:10.1016/j.jpeds.2012.06.050.

Lewis, M. H., Goldenberg, A., Anderson, R., Rothwell, E., \& Botkin, J. (2011). State laws regarding the retention and use of residual newborn screening blood samples. Pediatrics, 127(4), 703-712. doi:10. 1542/peds. 2010-1468.

McHugh, D., et al. (2011). Clinical validation of cutoff target ranges in newborn screening of metabolic disorders by tandem mass spectrometry: a worldwide collaborative project. Genetics in Medicine, 13(3), 230-254. doi:10.1097/GIM.0b013e31820d5e67.

National Newborn Screening and Global Resource Center (NNSGRC). (2006). National Newborn Screening 2006 Incidence Report. http:// genes-r-us.uthscsa.edu/sites/genes-r-us/files/resources/genetics/ 2006datareport.pdf.

National Newborn Screening and Global Resource Center (NNSGRC). (2011). Conditions screened by US programs: national newborn screening status report. Retrieved from website: http://genes-r-us. uthscsa.edu/sites/genes-r-us.uthscsa.edu/files/nbsdisorders.pdf.

Rose, S. R., \& Brown, R. S. (2006). Update of newborn screening and therapy for congenital hypothyroidism. Pediatrics, 117(6), 2290 2303. doi:10.1542/peds. 2006-0915.

Schoen, E. J., Baker, J. C., Colby, C. J., \& To, T. T. (2002). Cost-benefit analysis of universal tandem mass spectrometry for newborn screening. Pediatrics, $110(4), 781-786$. doi:10.1542/peds.110.4.781.

Szinnai, G. (2014). Genetics of normal and abnormal thyroid development in humans. Best Practice \& Research Clinical Endocrinology \& Metabolism, 28(2), 133-150. doi:10.1016/j.beem.2013.08.005.

U.S. Preventive Services Task Force. (1996). Screening for congenital hypothyroidism. In: Guide to clinical preventive services. 2nd ed. Washington, DC: U.S. Department of Health and Human Services; 503-507.

U.S. Preventive Services Task Force - Independent Expert Panel. (2008). Screening for congenital hypothyroidism: U.S. Preventive Services Task Force reaffirmation recommendation statement. NGC:006354 